\title{
DYNAMICS OF TWO-STRAIN INFLUENZA WITH ISOLATION AND PARTIAL CROSS-IMMUNITY*
}

\author{
M. NUÑO ${ }^{\dagger}$, Z. FENG ${ }^{\ddagger}$, M. MARTCHEVA§${ }^{\S}$ AND C. CASTILLO-CHAVEZ
}

\begin{abstract}
The time evolution of the influenza A virus is linked to a nonfixed landscape driven by interactions between hosts and competing influenza strains. Herd-immunity, cross-immunity, and age-structure are among the factors that have been shown to support strain coexistence and/or disease oscillations. In this study, we put two influenza strains under various levels of (interference) competition. We establish that cross-immunity and host isolation lead to periodic epidemic outbreaks (sustained oscillations) in this multistrain system. We compute the isolation reproductive number for each strain $\left(\Re_{i}\right)$ independently, as well as for the full system $\left(\Re_{q}\right)$, and show that when $\Re_{q}<1$, both strains die out. Subthreshold coexistence driven by cross-immunity is possible even when the isolation reproductive number of one strain is below 1 . Conditions that guarantee a winning type or coexistence are established in general. Oscillatory coexistence is established via Hopf bifurcation theory and confirmed via numerical simulations.
\end{abstract}

Key words. influenza, multiple strains, cross-immunity, isolation, stability, bifurcation, oscillations, coexistence

AMS subject classifications. 92D30, 92D25, 34C25, 34C60

DOI. $10.1137 /$ S003613990343882X

1. Introduction. Several studies have focused on the identification of mechanisms capable of supporting multiple-strain coexistence for diseases that provide permanent or temporary immunity $[19,18]$. Although there is still limited understanding on the role of cross-immunity (form of interference competition) between strains of a given virus, host variability (behavioral and immunological) is known to play a key role in maintaining virus diversity. Influenza epidemics and pandemics are closely linked to two types of mechanisms that maintain viral genetic diversity: antigenic "drift," the driver of strain heterogeneity, and antigenic "shift," the generator of subtype variability [28].

In 1918, the "Spanish Flu" pandemic caused the largest number of flu-related deaths worldwide in a single season [28]. More than 500,000 people died in the United States with 20-50 millions deaths worldwide. The "Asian Flu," a result of an antigenic shift in the hemmaglutinin and neuraminidase surface proteins, was responsible for about 70,000 deaths in the United States in 1969 [9]. The most recent and least lethal "pandemic," the "Hong Kong" pandemic, is attributed to the appearance of the H3N2 subtype [9].

* Received by the editors December 17, 2003; accepted for publication (in revised form) August 11, 2004; published electronically March 31, 2005. The research of the second and third authors was supported by NSF grants DMS-0314575, DMS-0137687, and DMS-0406119. The research of the third and fourth authors was supported with grants directed toward the Mathematical and Theoretical Biological Institute (MTBI) by the following institutions: National Science Foundation (NSF), National Security Agency (NSA), and Alfred P. Sloan Foundation.

http://www.siam.org/journals/siap/65-3/43882.html

$\dagger^{\dagger}$ Department of Biological Statistics and Computational Biology, Cornell University, Warren Hall, Ithaca, NY 14853-7801 (man16@cornell.edu).

${ }^{\ddagger}$ Department of Mathematics, Purdue University, West Lafayette, IN 47907.

$\S$ Department of Mathematics, University of Florida, Gainesville, FL 32611-8105.

ฯ Department of Mathematics and Statistics, Arizona State University, Tempe, AZ 85287-1804. 
The main focus of this paper is on the identification of competitive outcomes (mediated by cross-immunity) that result from the interactions between two strains of influenza A in a population where sick individuals may be isolated. Single-strain susceptible-infected-quarantined-recovered (SIQR) models with vital dynamics can generate sustained oscillations $[15,20]$. The introduction of a second strain increases the competition for susceptibles, a process mediated by cross-immunity in our setting. Will such competition preclude the possibility of sustained multistrain oscillations? We show that coexistence of both strains in the oscillatory regime is not uncommon and that oscillatory dynamics are possible for reasonable values of influenza parameters $[17,11,28]$.

Our paper is structured as follows. Section 2 introduces the general two-strain model; section 3 carries out the local stability analysis of the disease-free state; section 4 shows that periodic solutions can arise via a Hopf bifurcation; section 5 illustrates our theoretical results; section 6 summarizes our findings and collects some conclusions and thoughts.

2. Two-strain model. Theoretical work on two-strain models that incorporate the effects of interference competition in the context of communicable diseases goes back (at least) to the work of Dietz [13]. His work has been extended in the context of influenza $[5,1,6]$. None of these extensions considered the role of isolation. The study of mechanisms capable of generating sustained oscillations in single-strain epidemic models has received some attention in the last decades [19, 18]. Feng [14] and Feng and Thieme [15] showed that the introduction of an isolation class, in an otherwise standard SIR epidemiological model, is enough to generate sustained oscillations in single-strain models, but the region of parameter space where such oscillations are possible is unrealistic. Castillo-Chavez et al. [5, 6] provide support for the hypothesis that age-structure (age-dependent survival) and cross-immunity are enough to generate multistrain sustained oscillations in two-strain models without isolation. Here we show that cross-immunity in a two-strain system with isolation classes generate sustained oscillations within a region of parameter space that is consistent with the "flu" [11, 29, 24]. Furthermore, we identify the dependence of these regions on crossimmunity levels. The description of the two-strain model requires the division of the population into ten different classes: susceptibles $(S)$, infected with strain $i\left(I_{i}\right.$, primary infection), isolated with strain $i\left(Q_{i}\right)$, recovered from strain $i\left(R_{i}\right.$, as a result of primary infection), infected with strain $i$ ( $V_{i}$, secondary infection), given that the population had recovered from strains $j \neq i$, and recovered from both strains $(W)$. The population is assumed to mix randomly, except that the mixing is impacted by the process of quarantine/isolation $[14,15,20,8]$. Using the flow diagram in Figure 1, we arrive at the model

$$
\begin{aligned}
\frac{d S}{d t} & =\Lambda-\sum_{i=1}^{2} \beta_{i} S \frac{\left(I_{i}+V_{i}\right)}{A}-\mu S, \\
\frac{d I_{i}}{d t} & =\beta_{i} S \frac{\left(I_{i}+V_{i}\right)}{A}-\left(\mu+\gamma_{i}+\delta_{i}\right) I_{i}, \\
\frac{d Q_{i}}{d t} & =\delta_{i} I_{i}-\left(\mu+\alpha_{i}\right) Q_{i}, \\
\frac{d R_{i}}{d t} & =\gamma_{i} I_{i}+\alpha_{i} Q_{i}-\beta_{j} \sigma_{i j} R_{i} \frac{\left(I_{j}+V_{j}\right)}{A}-\mu R_{i}, \quad j \neq i,
\end{aligned}
$$




$$
\begin{aligned}
\frac{d V_{i}}{d t} & =\beta_{i} \sigma_{i j} R_{j} \frac{\left(I_{i}+V_{i}\right)}{A}-\left(\mu+\gamma_{i}\right) V_{i}, \quad j \neq i \\
\frac{d W}{d t} & =\sum_{i=1}^{2} \gamma_{i} V_{i}-\mu W \\
A & =S+W+\sum_{i=1}^{2}\left(I_{i}+V_{i}+R_{i}\right),
\end{aligned}
$$

where A denotes the population of nonisolated individuals and $\frac{\beta_{i} S\left(I_{i}+V_{i}\right)}{A}$ models the rate at which susceptibles become infected with strain $i$. That is, the $i$ th $(i \neq j)$ incidence rate is assumed to be proportional to both the number of susceptibles and the available proportion of $i$-infectious individuals, $\frac{\left(I_{i}+V_{i}\right)}{A}$. The parameter $\sigma_{i j}$ is a measure of the cross-immunity provided by a prior infection with strain $i$ to exposure with strain $j(i \neq j)$. Data from epidemiological studies conducted in Houston and Seattle $[27,17]$ generate rough measures of cross-immunity. From these studies it is clear that $\sigma_{i j} \in[0,1]$. Model (1) includes the models in [5, 6]. The absence of the $Q$ classes in earlier work precludes the possibility of sustained oscillations (see $[5,6]$ ). Isolation classes are not introduced after the $V$-classes to simplify the analysis and because often symptoms are less severe in these classes.

3. Disease invasion and stability. System (1) can support four equilibria. Analysis of the local stability of the trivial equilibrium (absence of disease) helps identify conditions under which the "flu" can invade. We assume (sections 3 and 4 )

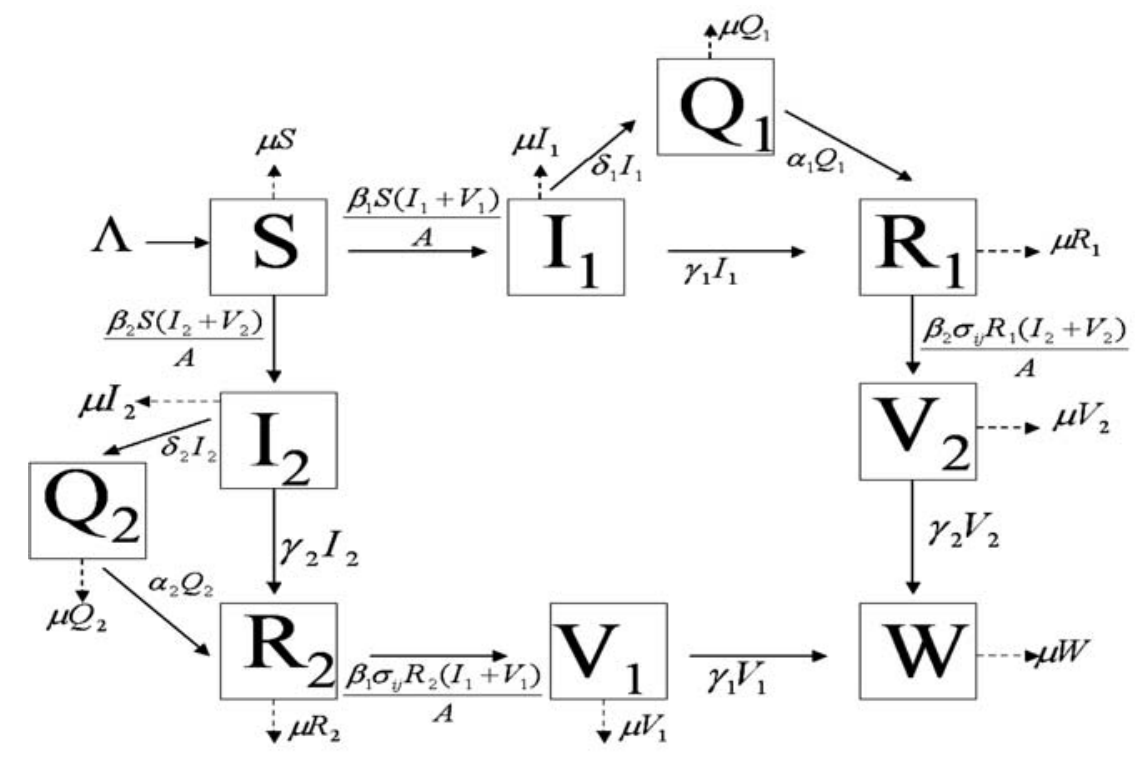

FIG. 1. Schematic diagram of disease dynamics when the host is exposed to two cocirculating strains. $\Lambda$ is the rate at which individuals are born into the population, $\beta_{i}$ denotes the transmission coefficient for strain $i, \mu$ is the per capita mortality rate, $\delta_{i}$ is the per capita isolation rate for strain $i, \gamma_{i}$ denotes the per capita recovery rate from strain $i, \alpha_{i}$ is the per capita rate at which individuals leave the isolated class as a result of infection with strain $i$, and $\sigma_{i j}$ is the relative susceptibility to strain $j$ for an individual previously infected with and recovered from strain $i(i \neq j) . \sigma_{i j}=0$ corresponds to total cross-immunity, while $\sigma_{i j}=1$ indicates no cross-immunity. 
that $\sigma_{12}=\sigma_{21}=\sigma$ (as it was done in $[5,6]$ ). This approach strongly limits the generality of our analysis, but the general case has turned out to be too difficult. The case where $\left|\sigma_{12}-\sigma_{21}\right|=\varepsilon$ (a small positive number) is explored numerically. Quantitative results do not seem to change when $\varepsilon$ is small enough. Flu-related mortality is low, and hence it is ignored. This is not a limiting factor over appropriate time scales. From our model (time scale for which demographic factors can be ignored)

$$
\frac{d}{d t} N=\Lambda-\mu N
$$

where $N=S+W+\sum_{i=1}^{2}\left(I_{i}+V_{i}+Q_{i}+R_{i}\right)$. Hence, $N(t) \rightarrow \frac{\Lambda}{\mu}$ as $t \rightarrow \infty$, and the results in [7] allow us to assume, without loss of generality, that $N(0)=\frac{\Lambda}{\mu}$. Hence, we set

$$
N(t) \equiv \frac{\Lambda}{\mu} \equiv S+W+\sum_{i=1}^{2}\left(I_{i}+V_{i}+Q_{i}+R_{i}\right)=A+Q \quad \text { for all } t,
$$

where $Q=Q_{1}+Q_{2}$ and $A=N-Q$.

The isolation reproductive number $\Re_{q}$, the average number of secondary infections generated by the simultaneous introduction of both strains in a fully susceptible population, is a function of the independent capacity of each strain to invade. Hence, $\Re_{q}=\max \left\{\Re_{1}, \Re_{2}\right\}$, where

$$
\Re_{i}=\frac{\beta_{i}}{\mu+\gamma_{i}+\delta_{i}} .
$$

Here, $\beta_{i}$ is the maximal effective transmission rate and $\left(\mu+\gamma_{i}+\delta_{i}\right)^{-1}$ is the average window of opportunity (effective infectious period) for transmission. It follows that $E_{0}$, the disease-free state, is locally asymptotically stable when $\Re_{q}<1$ and an unstable (saddle) whenever $\Re_{i}>1$ for either $i=1$ or $i=2$ (for details see Appendix A).

4. Nontrivial equilibria and sustained oscillations. Hethcote and Levin's 1989 survey of mathematical models [18], Feng [14], Feng and Thieme [15], Hethcote [19], and the recent comprehensive literature review of Hethcote and Levin [18] provide a solid perspective on what is known about the mechanisms that are capable of supporting sustained oscillations in epidemic models. Nonstructured two-strain SIR models with cross-immunity appear to be incapable of supporting them [1], but the addition of a third strain reverses the situation [26].

Here we focus on the role of isolation, a mechanism capable of generating sustained oscillations even in a single-strain model. The "flu" may survive in three states: either strain 1 or 2 survives or both strains coexist. Here, we carry only out the analysis in the symmetric cross-immunity case $\left(\sigma_{12}=\sigma_{21}=\sigma\right)$. We let $U=$ $\left(S, I_{1}, Q_{1}, R_{1}, V_{1}, I_{2}, Q_{2}, R_{2}, V_{2}, W\right)$ denote the state variables and focus on the analysis of the stability of the boundary equilibria, namely $E_{1}=\left(\tilde{S}, \tilde{I}_{1}, \tilde{Q}_{1}, \tilde{R}_{1}, 0,0,0,0,0,0\right)$. Setting $V_{1}=I_{2}=Q_{2}=R_{2}=V_{2}=W=0$ in (1) leads to the following relationships:

$$
\begin{array}{rlrl}
\frac{\tilde{S}}{\tilde{A}}=\frac{1}{\Re_{1}}, & & \frac{\tilde{I}_{1}}{\tilde{A}}=\mu\left(\mu+\alpha_{1}\right) \phi, \\
\frac{\tilde{Q}_{1}}{\tilde{A}}=\mu \delta_{1} \phi, & \frac{\tilde{R}_{1}}{\tilde{A}}=\left(\gamma_{1}\left(\mu+\alpha_{1}\right)+\alpha_{1} \delta_{1}\right) \phi,
\end{array}
$$


where

$$
\phi=\frac{\left(1-\frac{1}{\Re_{1}}\right)}{\left(\mu+\gamma_{1}\right)\left(\mu+\alpha_{1}\right)+\alpha_{1} \delta_{1}}
$$

and

$$
\tilde{A}=\frac{1}{\mu\left(1+\mu \delta_{1} \phi\right)}
$$

$E_{1}$ exists (entries are positive) and is unique if and only if $\Re_{1}>1$. Letting $A=N-Q$ and $S=A-\sum_{i=1}^{2}\left(I_{i}+V_{i}+R_{i}\right)-W$ allows the elimination of the $S$ equation. The $I_{i}$ equations become

$$
\frac{d I_{i}}{d t}=\beta_{i}\left(1-\frac{W+\sum_{i=1}^{2}\left(I_{i}+R_{i}+V_{i}\right)}{A}\right)\left(I_{i}+V_{i}\right)-\left(\mu+\gamma_{i}+\delta_{i}\right) I_{i} .
$$

The Jacobian at $E_{1}, \tilde{J}$, is given by the $9 \times 9$ (without $S$ ) matrix

$$
\tilde{J}=\left(\begin{array}{cccc}
G_{1} & * & * & * \\
0 & -\left(\mu+\gamma_{1}\right) & * & 0 \\
0 & 0 & G_{2} & 0 \\
0 & * & * & -\mu
\end{array}\right)
$$

where

$$
G_{1}=\left(\begin{array}{ccc}
-\beta_{1} \frac{\tilde{I}_{1}}{\tilde{A}} & -\beta_{1} \frac{\tilde{I}_{1}}{\tilde{A}}\left(1-\frac{1}{\Re_{1}}\right) & -\beta_{1} \frac{\tilde{I}_{1}}{\tilde{A}} \\
\delta_{1} & -\left(\mu+\alpha_{1}\right) & 0 \\
\gamma_{1} & \alpha_{1} & -\mu
\end{array}\right),
$$

and

$$
G_{2}=\left(\begin{array}{cccc}
\beta_{2} \frac{\tilde{S}}{\tilde{A}}-\left(\mu+\gamma_{2}+\delta_{2}\right) & 0 & 0 & \beta_{2} \frac{\tilde{S}}{\tilde{A}} \\
\delta_{2} & -\left(\mu+\alpha_{2}\right) & 0 & 0 \\
\gamma_{2} & \alpha_{2} & -\mu-\beta_{1} \sigma \frac{\tilde{I}_{1}}{\tilde{A}} & 0 \\
\beta_{2} \sigma \frac{\tilde{R}_{1}}{\tilde{A}} & 0 & 0 & \beta_{2} \sigma \frac{\tilde{R}_{1}}{\tilde{A}}-\left(\mu+\gamma_{2}\right)
\end{array}\right) .
$$

"*" represents a nonzero block matrix.

$G_{2}$ has two negative eigenvalues, plus the roots of the equation

$$
\lambda^{2}-c_{1} \lambda+c_{2}=0
$$

where

$$
\begin{aligned}
& c_{1}=\left(\mu+\gamma_{2}+\delta_{2}\right)\left(\frac{\Re_{2}-\Re_{1}}{\Re_{1}}\right)+\beta_{2} \sigma \frac{\tilde{R}_{1}}{\tilde{A}}-\left(\mu+\gamma_{2}\right), \\
& c_{2}=-\left(\mu+\gamma_{2}+\delta_{2}\right)\left[\beta_{2} \sigma \frac{\tilde{R}_{1}}{\tilde{A}}+\left(\mu+\gamma_{2}\right)\left(\frac{\Re_{2}-\Re_{1}}{\Re_{1}}\right)\right] .
\end{aligned}
$$

Hence $c_{1}<0$ and $c_{2}>0$ guarantee the local asymptotic stability (l.a.s.) of $E_{1}$.

$$
c_{1}<0 \Longleftrightarrow F_{1}\left(\Re_{1}, \Re_{2}\right):=\left(\mu+\gamma_{2}+\delta_{2}\right)\left(\frac{\Re_{2}}{\Re_{1}}-1+\sigma \Re_{2} \frac{\tilde{R}_{1}}{\tilde{A}}\right)-\left(\mu+\gamma_{2}\right)<0,
$$




$$
c_{2}>0 \Longleftrightarrow F_{2}\left(\Re_{1}, \Re_{2}\right):=\sigma\left(\mu+\gamma_{2}+\delta_{2}\right) \Re_{2} \frac{\tilde{R}_{1}}{\tilde{A}}+\left(\mu+\gamma_{2}\right)\left(\frac{\Re_{2}}{\Re_{1}}-1\right)<0,
$$

where $\tilde{R}_{1} / \tilde{A}$ is given in (2). In the case of full immunity $(\sigma=0)$, the conditions in (6) and (7) hold if and only if $\Re_{2}<\Re_{1}$. That is, when $\sigma=0, E_{1}$ has l.a.s., as long as $\Re_{1}>1$ and $\frac{\Re_{1}}{\Re_{2}}>1$ and $E_{1}$ is unstable $(\sigma=0)$ when $\frac{\Re_{2}}{\Re_{1}}>1$. As cross-immunity between strains diminishes $(0<\sigma \uparrow 1)$, alternative conditions are needed to ensure that (6) and (7) hold. To find these conditions we rewrite $F_{1}$ in terms of $F_{2}$,

$$
F_{1}\left(\Re_{1}, \Re_{2}\right)=F_{2}\left(\Re_{1}, \Re_{2}\right)+\delta_{2}\left(\frac{\Re_{2}}{\Re_{1}}-1\right)-\left(\mu+\gamma_{2}\right),
$$

and observe that $F_{1} \leq F_{2}$ when $\Re_{2}<\Re_{1}$. Alternatively, the introduction of

$$
f\left(\Re_{1}\right) \equiv \frac{\Re_{1}}{1+\sigma\left(\Re_{1}-1\right)\left(1+\frac{\delta_{2}}{\mu+\gamma_{2}}\right)\left(1-\frac{\mu\left(\mu+\alpha_{1}\right)}{\left(\mu+\gamma_{1}\right)\left(\mu+\alpha_{1}\right)+\alpha_{1} \delta_{1}}\right)}
$$

implies that $F_{2}<0$ if and only if $\Re_{2}<f\left(\Re_{1}\right)\left(0<f\left(\Re_{1}\right)<\Re_{1}\right)$. Therefore, $\Re_{2}<f\left(\Re_{1}\right)$ implies that $F_{1} \leq F_{2}<0$. Hence, all eigenvalues of $G_{2}$ have negative real part when $\frac{\Re_{2}}{f\left(\Re_{1}\right)}<1$, and $E_{1}$ is unstable when $\frac{\Re_{2}}{f\left(\Re_{1}\right)}>1$. Similarly, the use of

$$
g\left(\Re_{2}\right) \equiv \frac{\Re_{2}}{1+\sigma\left(\Re_{2}-1\right)\left(1+\frac{\delta_{1}}{\mu+\gamma_{1}}\right)\left(1-\frac{\mu\left(\mu+\alpha_{2}\right)}{\left(\mu+\gamma_{2}\right)\left(\mu+\alpha_{2}\right)+\alpha_{2} \delta_{2}}\right)}
$$

implies that the eigenvalues corresponding to the system when strain 2 has become established are all negative whenever $\Re_{1}<g\left(\Re_{2}\right)$. The boundary endemic equilibria for strain $2\left(E_{2}\right)$ are stable when $\frac{\Re_{1}}{g\left(\Re_{2}\right)}<1$ and unstable when $\frac{\Re_{1}}{g\left(\Re_{2}\right)}>1$.

Conditions that guarantee "coexistence" equilibria are formulated in terms of the (conditional) "invasion" reproductive numbers for strains 2 and $1\left(\Re_{2}^{1}\right.$ and $\left.\Re_{1}^{2}\right)$. $\Re_{2}^{1}$ is defined as the number of secondary infections generated by a "typical" strain-2infected individual in a population where strain 1 is endemic $\left(E_{1}\right)$. From conditions (6) and (7) (which ensure the stability of $E_{1}$ ) we find that

$$
\Re_{2}^{1}=\frac{\beta_{2}}{\mu+\gamma_{2}+\delta_{2}} \frac{\tilde{S}}{\tilde{A}}+\frac{\beta_{2} \sigma}{\mu+\gamma_{2}} \frac{\tilde{R}_{1}}{\tilde{A}} .
$$

Similarly, the (conditional) invasion reproductive number of strain 1 under the assumption that strain 2 is endemic is given by

$$
\Re_{1}^{2}=\frac{\beta_{1}}{\mu+\gamma_{1}+\delta_{1}} \frac{\tilde{S}}{\tilde{A}}+\frac{\beta_{1} \sigma}{\mu+\gamma_{1}} \frac{\tilde{R}_{2}}{\tilde{A}} .
$$

The condition $\Re_{2}<f\left(\Re_{1}\right)$ is equivalent to the condition $\Re_{2}^{1}<1$, while the condition $\Re_{1}<g\left(\Re_{2}\right)$ is equivalent to the condition $\Re_{1}^{2}<1 . \Re_{i}^{j}(i, j=1,2 i \neq j)$ is in fact the result of two additive contributions: $\beta_{i} /\left(\mu+\gamma_{i}+\delta_{i}\right)$ gives the number of secondary cases that a "typical" strain- $i$-infected individual will generate in the fully susceptible proportion of the population $\tilde{S} / \tilde{A}$, while $\beta_{i} \sigma /\left(\mu+\gamma_{i}\right)$ is the number of secondary cases that a "typical" strain- $i$-infected individual will generate in the "cross-immune" proportion of the susceptible population $\tilde{R}_{i} / \tilde{A}$. Note that whenever $\Re_{i}>1$ and $\Re_{j}^{i}<1$, the boundary equilibrium $E_{i}$ is locally stable. 
4.1. Multiple and subthreshold coexistence. Both strains coexist if their basic reproductive numbers are above 1 (see Figures 2(a), (b), (d)), but subthreshold coexistence is possible (see Figure 2(c)). In order to see this (in system (1)) let $S / A=s, I_{i} / A=i_{i}, Q_{i} / A=q_{i}, R_{i} / A=r_{i}, V_{i} / A=v_{i}, W / A=w$, and $n=\frac{N}{A}$ with $\sigma_{12}=\sigma_{21}=\sigma$. The equilibrium conditions for the rescaled system are

$$
\begin{aligned}
\beta_{1} s\left(i_{1}+v_{1}\right)+\beta_{2} s \frac{\Re_{1}}{\Re_{2}} \frac{i_{2}}{i_{1}}\left(i_{1}+v_{1}\right)+\mu s & =\mu\left(1+\eta_{1} i_{1}+\eta_{2} i_{2}\right), \\
\beta_{1} s\left(i_{1}+v_{1}\right) & =\left(\mu+\gamma_{1}+\delta_{1}\right) i_{1}, \\
\frac{\left(i_{1}+v_{1}\right)}{\left(i_{2}+v_{2}\right)} & =\frac{\Re_{2}}{\Re_{1}} \frac{i_{1}}{i_{2}}, \\
\beta_{2} \sigma r_{1} \frac{\Re_{1}}{\Re_{2}} \frac{i_{2}}{i_{1}}\left(i_{1}+v_{1}\right)+\mu r_{1} & =\left(\gamma_{1}+\kappa_{1}\right) i_{1}, \\
\beta_{1} \sigma r_{2}\left(i_{1}+v_{1}\right) & =\left(\mu+\gamma_{1}\right) v_{1}, \\
\beta_{1} \sigma r_{2}\left(i_{1}+v_{1}\right)+\mu r_{2} & =\left(\gamma_{2}+\kappa_{2}\right) i_{2}, \\
\beta_{2} \sigma r_{1} \frac{\Re_{1}}{\Re_{2}} \frac{i_{2}}{i_{1}}\left(i_{1}+v_{1}\right)+\left(\mu+\gamma_{2}\right) i_{2} & =\left(\mu+\gamma_{2}\right)\left(i_{2}+v_{2}\right),
\end{aligned}
$$

where

$$
\begin{aligned}
\kappa_{i} & =\frac{\alpha_{i} \delta_{i}}{\mu+\alpha_{i}}, \\
\eta_{i} & =\frac{\delta_{i}}{\mu+\alpha_{i}} .
\end{aligned}
$$

Expressions (10) and (11) can be solved for $s$, that is,

$$
s=\frac{\mu\left(1+\eta_{1} i_{1}+\eta_{2} i_{2}\right)}{\beta_{1}\left(i_{1}+v_{1}\right)+\beta_{2}\left(i_{2}+v_{2}\right)+\mu}=\frac{\left(\mu+\gamma_{1}+\delta_{1}\right) i_{1}}{\beta_{1}\left(i_{1}+v_{1}\right)} .
$$

From (17) it follows that

$$
\frac{\mu \beta_{1}\left(i_{1}+v_{1}\right)\left(1+\eta_{1} i_{1}+\eta_{2} i_{2}\right)}{\beta_{1}\left(i_{1}+v_{1}\right)+\beta_{2}\left(i_{2}+v_{2}\right)+\mu}=\left(\mu+\gamma_{1}+\delta_{1}\right) i_{1} .
$$

Equation (14) and its symmetric analogue are solved for $r_{1}$ and $r_{2}$. In fact,

$$
r_{1}=\frac{\left(\mu+\gamma_{2}\right) v_{2}}{\beta_{2} \sigma\left(i_{2}+v_{2}\right)}
$$

and

$$
r_{2}=\frac{\left(\mu+\gamma_{1}\right) v_{1}}{\beta_{1} \sigma\left(i_{1}+v_{1}\right)}
$$

From (13) and the relationship $\beta_{2} \sigma\left(i_{2}+v_{2}\right) r_{1}=\left(\mu+\gamma_{2}\right) v_{2}$ we have that

$$
\left(\gamma_{1}+\kappa_{1}\right) i_{1}-\left(\mu+\gamma_{2}\right) v_{2}=\mu r_{1} .
$$

Using (12) and substituting the above expression for $r_{1}$ helps rewrite (13) as

(19) $\left(\gamma_{1}+\kappa_{1}\right) i_{1}-\left(\mu+\gamma_{2}\right) \frac{\Re_{1} i_{2}\left(i_{1}+v_{1}\right)}{\Re_{2} i_{1}}+\left(\mu+\gamma_{2}\right) i_{2}=\frac{\mu\left(\mu+\gamma_{2}\right)}{\beta_{2} \sigma}-\frac{\mu\left(\mu+\gamma_{2}\right) i_{2}}{\beta_{2} \sigma\left(i_{2}+v_{2}\right)}$. 
Solving for $\left(i_{2}+v_{2}\right)$ in (13) and using (19) leads to

$$
\left(\gamma_{1}+\kappa_{1}\right) i_{1}-\left(\mu+\gamma_{2}\right) \frac{\Re_{1} i_{2}\left(i_{1}+v_{1}\right)}{\Re_{2} i_{1}}+\left(\mu+\gamma_{2}\right) i_{2}=\frac{\mu\left(\mu+\gamma_{2}\right)}{\beta_{2} \sigma}\left(1-\frac{\Re_{2} i_{1}}{\Re_{1}\left(i_{1}+v_{1}\right)}\right) .
$$

In a similar manner, using $r_{2}$ and the expression $\beta_{1} \sigma r_{2}\left(i_{1}+v_{1}\right)=\left(\mu+\gamma_{1}\right) v_{1}$ leads to the reduced system

$$
\begin{aligned}
\frac{\mu \beta_{1}\left(1+\eta_{1} i_{1}+\eta_{2} i_{2}\right)\left(i_{1}+v_{1}\right)}{\beta_{1}\left(i_{1}+v_{1}\right)+\frac{\beta_{2} \Re_{1}\left(i_{1}+v_{1}\right) i_{2}}{\Re_{2} i_{1}}+\mu} & =\left(\mu+\gamma_{1}+\delta_{1}\right) i_{1}, \\
\left(\gamma_{1}+\kappa_{1}\right) i_{1}-\left(\mu+\gamma_{2}\right) i_{2}\left(\frac{\Re_{1}\left(i_{1}+v_{1}\right)}{\Re_{2} i_{1}}-1\right) & =\frac{\mu\left(\mu+\gamma_{2}\right)}{\beta_{2} \sigma}\left(1-\frac{\Re_{2} i_{1}}{\Re_{1}\left(i_{1}+v_{1}\right)}\right), \\
\left(\gamma_{2}+\kappa_{2}\right) i_{2}-\left(\mu+\gamma_{1}\right) v_{1} & =\frac{\mu\left(\mu+\gamma_{1}\right) v_{1}}{\beta_{1} \sigma\left(i_{1}+v_{1}\right)} .
\end{aligned}
$$

From the first equation in (17) we get that

$$
\begin{aligned}
\left(i_{1}+v_{1}\right) & =\frac{\mu\left(\mu+\gamma_{1}+\delta_{1}\right) i_{1}}{\beta_{1}\left[\mu\left(1+\eta_{1} i_{1}+\eta_{2} i_{2}\right)-i_{1}\left(\mu+\gamma_{1}+\delta_{1}\right)-i_{2}\left(\mu+\gamma_{2}+\delta_{2}\right)\right]}, \\
& =\frac{\mu\left(\mu+\gamma_{1}+\delta_{1}\right) i_{1}}{\beta_{1}\left[\triangle\left(i_{1}, i_{2}\right)\right]},
\end{aligned}
$$

where

$$
\triangle\left(i_{1}, i_{2}\right)=\mu\left(1+\eta_{1} i_{1}+\eta_{2} i_{2}\right)-i_{1}\left(\mu+\gamma_{1}+\delta_{1}\right)-i_{2}\left(\mu+\gamma_{2}+\delta_{2}\right) .
$$

The substitution of (22) into the second equation in (21) and the use of its symmetric analogue gives a system of equations (in terms of $i_{1}$ and $i_{2}$ only). The system is

$$
\begin{aligned}
& \left(\triangle\left(i_{1}, i_{2}\right)\right)^{2} \frac{\left(\mu+\gamma_{2}\right) \Re_{2}}{\beta_{2} \sigma}+\triangle\left(i_{1}, i_{2}\right)\left[\left(\gamma_{1}+\kappa_{1}\right) i_{1}+\left(\mu+\gamma_{2}\right) i_{2}-\frac{\mu\left(\mu+\gamma_{2}\right)}{\beta_{2} \sigma}\right] \\
& -\frac{\mu\left(\mu+\gamma_{2}\right) i_{2}}{\Re_{2}}=0, \\
& \left(\triangle\left(i_{1}, i_{2}\right)\right)^{2} \frac{\left(\mu+\gamma_{1}\right) \Re_{1}}{\beta_{1} \sigma}+\triangle\left(i_{1}, i_{2}\right)\left[\left(\gamma_{2}+\kappa_{2}\right) i_{2}+\left(\mu+\gamma_{1}\right) i_{1}-\frac{\mu\left(\mu+\gamma_{1}\right)}{\beta_{1} \sigma}\right] \\
& -\frac{\mu\left(\mu+\gamma_{1}\right) i_{1}}{\Re_{1}}=0 .
\end{aligned}
$$

Positive solutions of (23) are only candidates for coexistence equilibria, as we must check that the corresponding values $\left(s, q_{i}, r_{i}, v_{i}\right.$, and $\left.w\right)$ are positive. Numerical simulations show that such solutions exist in the ranges $0 \leq i_{1} \leq 1$ and $0 \leq i_{2} \leq 1$ for parameter values that are reasonable for the "flu." Figure 2 (a) $\left(\Re_{1}>1\right.$ and $\left.\Re_{2}>1\right)$ shows one such intersection in the positive quadrant. Subthreshold coexistence equilibrium is also possible for $\Re_{1}<1$ and $\Re_{2}>1$ (see Figure 2(c)). As we increase the basic reproductive number of both strains and allow varying levels of cross-immunity $(\sigma=0.5$ and $\sigma=0.6)$, Figures 2(b) and 2(d) show that two intersections (in the positive quadrant) are possible. That is, multiple coexistence equilibria exist (it was verified that all classes are positive). This possibility is absent from prior influenza models. For the parameter values in Table 1, only a single coexistence equilibrium is biologically reasonable. Figures 2(a), (c) provide additional examples where coexistence and subthreshold coexistence are possible. 

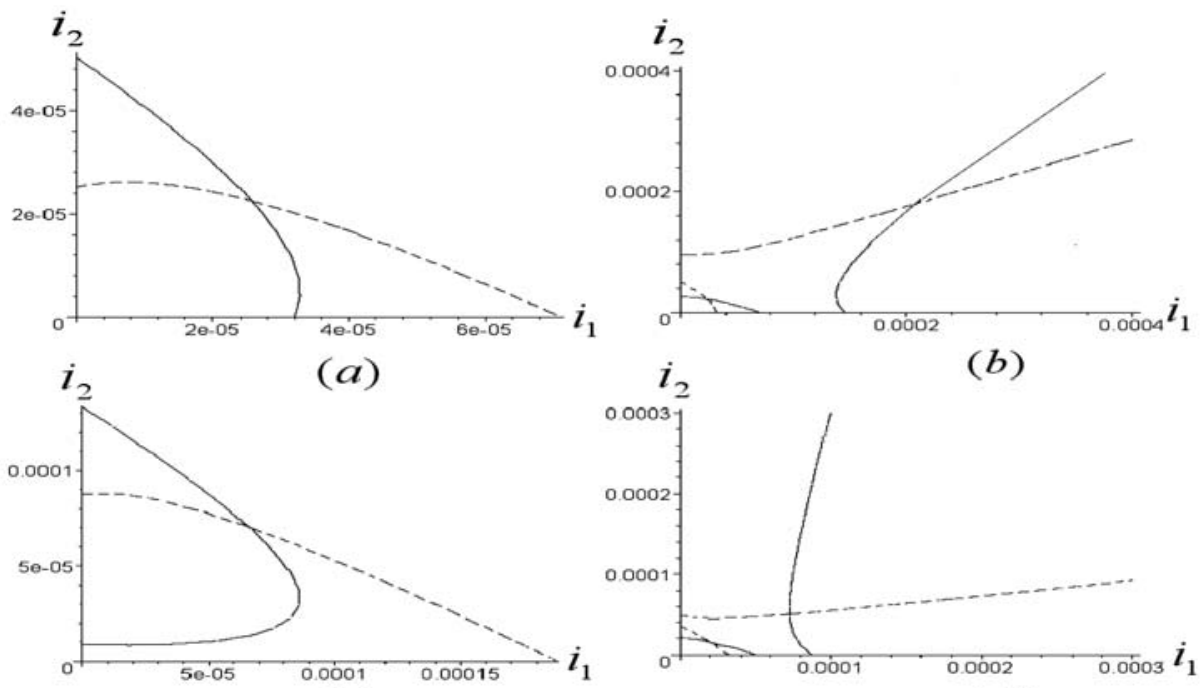

(c)

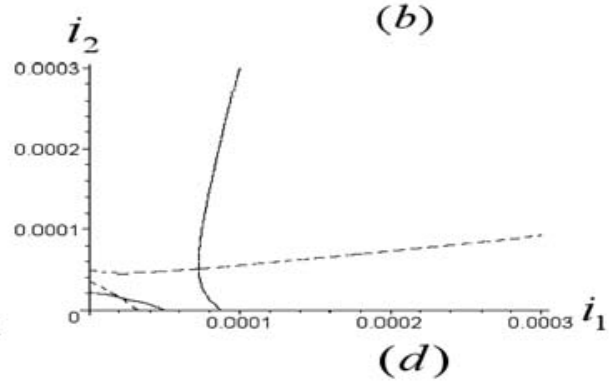

FIG. 2. Positive solutions of (23) are provided to illustrate the existence of multiple endemic states and subthreshold coexistence (section 4.1). The horizontal axis is the fraction $\left(i_{1}=I_{1} / A\right)$ of individuals infected with strain 1 , and the vertical axis depicts the fraction $\left(i_{2}=I_{2} / A\right)$ of individuals infected with strain 2. (a) $\Re_{1}=2, \Re_{2}=1.99$, and $\sigma=0.9$. (b) $\Re_{1}=2, \Re_{2}=1.99$, and $\sigma=0.5$. (c) $\Re_{1}=0.75, \Re_{2}=2.9$, and $\sigma=0.9$. (d) $\Re_{1}=2.83, \Re_{2}=2.93$, and $\sigma=0.6$.

TABLE 1

The parameter values used for the numerical simulations are provided here. The initial conditions are given by $s(0)=0.4, i_{1}(0)=0.199, r_{1}(0)=r_{2}(0)=0.2$, and $i_{2}(0)=q_{1}(0)=q_{2}=w(0)=0$ (only one strain present initially) and $s(0)=0.4, i_{1}(0)=0.199, q_{1}(0)=0.1, r_{1}(0)=r_{2}(0)=0.2$, $i_{2}(0)=0.001$, and $q_{1}(0)=q_{2}(0)=w(0)=0$ (both strains present initially).

\begin{tabular}{||l|l|l||}
\hline Parameters & Definition & Values \\
\hline$\Re_{i}$ & $\begin{array}{l}\text { Number of secondary cases generated by a primary case infected } \\
\text { with strain } i\end{array}$ & $(0.75,4.5)$ \\
& Cross-immunity against strain $j$ following an infection & $(0.008,0.8)$ \\
$\Lambda$ & with strain $i$ & \\
$\sigma_{i}$ & Rate at which individuals are born into the population & 0.00004 \\
$\delta_{i}$ & Rate at which individuals leave isolation $\left(1 / \alpha_{i}=\right.$ days $)$ & $(1,15)$ \\
$\gamma_{i}$ & Rate of isolation with strain $i, i=1,2\left(1 / \delta_{i}=\right.$ days $)$ & $(1,6)$ \\
$\beta_{i}$ & Recovery rate from strain $i, i=1,2\left(1 / \gamma_{i}=\right.$ days $)$ & $(5,7)$ \\
$\mu$ & Transmission coefficient for strain $i, i=1,2$ & $(0.4,2.2)$ \\
\end{tabular}

4.2. Sustained oscillations. A detailed study of the nature of the eigenvalues of matrix $G_{1}$ makes use of the identity

$$
\beta_{1} \frac{\tilde{S}}{\tilde{A}}-\left(\mu+\gamma_{1}+\alpha_{1}\right)=-\beta_{1} \frac{\tilde{I}_{1}}{\tilde{A}}
$$

and the fact that $\Re_{1}$ does not depend on $\alpha_{1}$. The dependence of $f$ on $\alpha_{1}$ is in the order of $\mu$, and this observation is used in the study of the characteristic equation associated with $G_{1}$ as we search for the possibility of sustained oscillations. The characteristic 
equation is

$$
\omega^{3}+a_{1} \omega^{2}+a_{2} \omega+a_{3}=0
$$

where

$$
\begin{aligned}
& a_{1}=2 \mu+\alpha_{1}+\Re_{1}\left(\mu+\gamma_{1}+\delta_{1}\right) \mu\left(\mu+\alpha_{1}\right) \phi, \\
& a_{2}=\mu\left(\mu+\alpha_{1}\right)\left[1+\Re_{1}\left(\mu+\gamma_{1}+\delta_{1}\right)\left(2 \mu+\alpha_{1}+\gamma_{1}+\delta_{1}\left(1-\frac{1}{\Re_{1}}\right)\right) \phi\right], \\
& a_{3}=\left[\mu^{2}+\alpha_{1} \mu+\delta_{1} \alpha_{1}+\gamma_{1} \mu+\gamma_{1} \alpha_{1}+\delta_{1} \mu\left(1-\frac{1}{\Re_{1}}\right)\right] \Re_{1}\left(\mu+\gamma_{1}+\delta_{1}\right) \mu\left(\mu+\alpha_{1}\right) \phi .
\end{aligned}
$$

Since $a_{1}, a_{2}$, and $a_{3}$ are all positive $\left(\Re_{1}>1\right)$, then the cubic equation in (24) has either three negative or one negative root and possibly two complex conjugate roots. Differences in epidemiological and demographic time scales are used to tease out the nature of the roots of $(24)$. The average life expectancy $(1 / \mu)$ is in the order of decades, while the infective $\left(1 / \delta_{i}\right.$ or $\left.1 / \gamma_{i}\right)$ and isolation periods $\left(1 / \alpha_{i}\right)$ are just a few days. That is, $\mu$ is much smaller than $\delta_{i}, \gamma_{i}$, and $\alpha_{i}$. Following early approaches $[14,15,23]$, we carry out an asymptotic expansion on the coefficients of (24) using $\mu$. From (25), it is clear that $a_{i}$ are analytic functions of $\mu>-\varepsilon$ for some $\varepsilon>0$. Hence,

$$
\begin{aligned}
& a_{1}=\alpha_{1}+\left(\Re_{1}^{*}+1\right) \mu+O\left(\mu^{2}\right), \\
& a_{2}=\left[\left(\alpha_{1}+\gamma_{1}\right) \Re_{1}^{*}-\gamma_{1}+\frac{\delta_{1}\left(\Re_{1}^{*}-1\right)^{2}}{\Re_{1}^{*}}\right] \mu+O\left(\mu^{2}\right), \\
& a_{3}=\alpha_{1}\left(\delta_{1}+\gamma_{1}\right)\left(\Re_{1}^{*}-1\right) \mu+O\left(\mu^{2}\right),
\end{aligned}
$$

where $\Re_{1}^{*}$ denotes $\Re_{1}(\mu)$ evaluated at $\mu=0$, that is, $\Re_{1}^{*}=\Re_{1}(0)$. The continuous dependence of the roots on $\mu$ is acknowledged by letting $\omega_{i}=\omega_{i}(\mu)(i=1,2,3)$ denote the roots of (24) (for a fixed value of $\mu$ ). In the limiting case, $\mu=0, a_{2}$ and $a_{3}$ are zero, while $a_{1}=\alpha_{1}$ (from (26)). The characteristic polynomial in this limiting case is simply

$$
\omega^{3}+\alpha_{1} \omega^{2}=0
$$

which has the roots $\omega_{1}(0)=-\alpha_{1}$ and $\omega_{2}(0)=\omega_{3}(0)=0$. Hence, by continuity, $\omega_{1}(\mu)=-\alpha_{1}+O(\mu)$ is a negative real root of (24) for small $\mu>0$. In order to use arguments similar to those found in [14, 15, 23] or in Kato [23, II, $\S 1$, section 2], it is assumed that the roots $\omega_{2}(\mu)$ and $\omega_{3}(\mu)$ have expansions of the form

$$
\omega(\mu)=\sum_{j=1}^{\infty} \xi_{j} \nu^{j}, \quad \nu=\mu^{\frac{1}{2}} .
$$

The formal substitution of (27) into (24) (neglecting higher-order terms in $\nu$ ) yields

$$
\begin{aligned}
& {\left[\xi_{1}^{2} \alpha_{1}+\alpha_{1}\left(\delta_{1}+\gamma_{1}\right)\left(\Re_{1}^{*}-1\right)\right] \nu^{2}} \\
& \quad+\left[\xi_{1}^{3}+2 \xi_{1} \xi_{2} \alpha_{1}+\left(\left(\gamma_{1}+\alpha_{1}\right) \Re_{1}^{*}-\gamma_{1}+\frac{\delta_{1}\left(\Re_{1}^{*}-1\right)^{2}}{\Re_{1}^{*}}\right) \xi_{1}\right] \nu^{3}+O\left(\nu^{4}\right)=0 .
\end{aligned}
$$

Hence,

$\xi_{1}^{2}=-\left(\gamma_{1}+\delta_{1}\right)\left(\Re_{1}^{*}-1\right) \quad$ and $\quad \xi_{2}=-\frac{1}{2 \alpha_{1}}\left(\xi_{1}^{2}-\gamma_{1}+\left(\gamma_{1}+\alpha_{1}\right) \Re_{1}^{*}+\frac{\delta_{1}\left(\Re_{1}^{*}-1\right)^{2}}{\Re_{1}^{*}}\right)$. 
From the fact that $\Re_{1}^{*}>1$ we have that

$$
\xi_{1}= \pm i \sqrt{\left(\gamma_{1}+\delta_{1}\right)\left(\Re_{1}^{*}-1\right)} \quad \text { and } \quad \xi_{2}=-\frac{1}{2 \alpha_{1}}\left(\alpha_{1} \Re_{1}^{*}+\delta_{1}\left(\frac{1}{\Re_{1}^{*}}-1\right)\right) .
$$

That is, the three roots of (24) have expressions of the form

$$
\omega_{1}(\nu)=-\alpha_{1}+O\left(\nu^{2}\right)
$$

and

$$
\omega_{2,3}(\nu)= \pm i\left(\left(\gamma_{1}+\delta_{1}\right)\left(\Re_{1}^{*}-1\right)\right)^{\frac{1}{2}} \nu-\frac{1}{2 \alpha_{1}}\left(\alpha_{1} \Re_{1}^{*}+\delta_{1}\left(\frac{1}{\Re_{1}^{*}}-1\right)\right) \nu^{2}+O\left(\nu^{3}\right) .
$$

We select $\alpha_{1}$ as our implicit bifurcation parameter $\left(1 / \alpha_{1}\right.$ is the isolation period for strain 1). We observe that $\alpha_{1}=\alpha_{1}(\nu)$ is a function of $\nu$ that satisfies the equation $\xi_{2}\left(\alpha_{1}(0)\right)=0$. Hence

$$
\alpha_{1}(0)=\frac{\delta_{1}}{\Re_{1}^{*}}\left(1-\frac{1}{\Re_{1}^{*}}\right) .
$$

The use of functions $\omega_{2,3}=\omega_{2,3}\left(\alpha_{1}, \nu\right)$ and $H\left(\alpha_{1}, \nu\right)=\frac{1}{\nu^{2}} \mathbb{R} \omega_{2,3}\left(\alpha_{1}, \nu\right)$ (where $\mathbb{R} \omega_{2,3}\left(\alpha_{1}, \nu\right)$ denotes the real part of the roots of (24) as given in (30)) imply that $H\left(\alpha_{1}(0), 0\right)=\xi_{2}\left(\alpha_{1}(0)\right)=0$. The implicit function theorem guarantees the existence of a critical function $\alpha_{c}(\nu)=\frac{\delta_{1}}{\Re_{1}^{*}}\left(1-\frac{1}{\Re_{1}^{*}}\right)+O(\nu)$, such that $H\left(\alpha_{c}(\nu), \nu\right)=0$ for small $\nu$. Clearly, $\alpha_{c}(\nu)>0$, as long as $\Re_{1}^{*}>1$. Furthermore, since

$$
\frac{\partial H}{\partial \alpha_{1}}(0)=-\frac{1}{2 \delta_{1}} \frac{\Re_{1}^{* 3}}{\left(\Re_{1}^{*}-1\right)}<0
$$

nonresonance holds [21], that is, as the frequency of strain 1 approaches that of strain 2 (or vice versa). Solutions remain bounded. The use of $\alpha_{1}$ as a bifurcation parameter shows that the roots $\omega_{2,3}$ cross the imaginary axis from left to right whenever $\alpha_{1}$ crosses $\alpha_{c}$ from right to left. That is, the crossing is transversal. Hence, a Hopf bifurcation occurs near the critical point $\alpha_{c}=\delta_{1}\left(\Re_{1}^{*}-1\right) /\left(\Re_{1}^{*}\right)^{2}$. We collect these results in the following theorem.

THEOREM 1. There are two functions: $f\left(\Re_{1}\right)$ as defined in $(8)$, and $\alpha_{c}(\mu)$ defined for small $\mu>0$ by

$$
\alpha_{c}(\mu)=\frac{\delta_{1}}{\Re_{1}^{*}}\left(1-\frac{1}{\Re_{1}^{*}}\right)+O\left(\mu^{\frac{1}{2}}\right),
$$

with the following properties: (i) The boundary endemic equilibrium $E_{1}$ is locally asymptotically stable if $\Re_{2}<f\left(\Re_{1}\right)$ and $\alpha_{1}<\alpha_{c}(\mu)$, and unstable if $\Re_{2}>f\left(\Re_{1}\right)$ or $\alpha_{1}>\alpha_{c}(\mu)$. (ii) When $\Re_{2}<f\left(\Re_{1}\right)$, periodic solutions arise at $\alpha_{1}=\alpha_{c}(\mu)$ via Hopf bifurcation for small enough $\mu>0$. The period can be approximated by

$$
T=\frac{2 \pi}{\left|\Im \omega_{2,3}\right|} \approx \frac{2 \pi}{\left(\left(\gamma_{1}+\delta_{1}\right)\left(\Re_{1}^{*}-1\right)\right)^{\frac{1}{2}} \mu^{\frac{1}{2}}}
$$

or (using (2)) by

$$
T \approx \frac{2 \pi}{\left(\gamma_{1}+\delta_{1}\right)^{\frac{1}{2}}\left(\frac{\hat{I_{1}}}{\hat{A}}\right)^{\frac{1}{2}} \mu^{\frac{1}{2}}},
$$


where $\hat{I}_{1} / \hat{A}$ denotes $\beta_{1} \tilde{I}_{1} / \mu \tilde{A}$ evaluated at $\mu=0$ and $\left|\Im \omega_{2,3}\right|$ refers to the imaginary roots calculated in $(30)$.

The latter expression for $T$ allows one to compare the period of this model with the quasi periods obtained from models which do not include an isolation class $[5,6]$. Since we have focused on the symmetric case, an analogous result for the second boundary equilibrium $E_{2}$ can be stated immediately. That is, the boundary endemic equilibrium $E_{2}$ is locally asymptotically stable if $\Re_{1}<g\left(\Re_{2}\right)$ and $\alpha_{2}<\alpha_{c}(\mu)$. It becomes unstable if $\Re_{1}>g\left(\Re_{2}\right)$ or $\alpha_{2}>\alpha_{c}(\mu)$. A summary of the stability results as presented in Theorem 1 for strain 1 is obtained for strain 2 by replacing the parameter indices 1's with 2's and replacing $f\left(\Re_{1}\right)$ with $g\left(\Re_{2}\right)$. Functions $f\left(\Re_{1}\right)$ and $g\left(\Re_{2}\right)$ help in the characterization of the stability and coexistence regions for strains 1 and 2. Changes in the regions of stability for either a single or for both strains can be illustrated as the coefficients of cross-immunity are varied. For instance, from (8) we can compute the value of $\sigma$ at which

$$
\left.f^{\prime}\left(\Re_{1}\right) \equiv \frac{\partial f\left(\Re_{1}, \sigma\right)}{\partial \Re_{1}}\right|_{\sigma_{1}^{*}}=0,
$$

namely

$$
\sigma_{1}^{*}=\frac{1}{\left(1+\frac{\delta_{2}}{\mu+\gamma_{2}}\right)\left(1-\frac{\mu\left(\mu+\alpha_{1}\right)}{\left(\mu+\gamma_{1}\right)\left(\mu+\alpha_{1}\right)+\alpha_{1} \delta_{1}}\right)} .
$$

Hence, for all $\Re_{1}>1$,

$$
f^{\prime}\left(\Re_{1}\right)>(<,=) 0, \quad f\left(\Re_{1}\right)>(<,=) 1 \quad \text { if } \sigma<(>,=) \sigma_{1}^{*} .
$$

These properties are easily verified, since (from (8))

$$
f\left(\Re_{1}\right)=\frac{\Re_{1}}{1+\frac{\sigma}{\sigma_{1}^{*}}\left(\Re_{1}-1\right)} \quad \text { and } \quad f^{\prime}\left(\Re_{1}\right)=\frac{1-\frac{\sigma}{\sigma_{1}^{*}}}{\left(1+\frac{\sigma}{\sigma_{1}^{*}}\left(\Re_{1}-1\right)\right)^{2}} .
$$

From the facts that $f\left(\Re_{1}\right)<\Re_{1}$ and $f(1)=1$ we see that Figure 3 captures the properties of the curve $\Re_{2}=f\left(\Re_{1}\right)$. Similar curve "boundary" features can be studied using threshold value $\sigma_{2}^{*}$ (interchanging the subscripts 1 and 2 in the expression of $\sigma_{1}^{*}$ ) and the function $\Re_{1}=g\left(\Re_{2}\right)$ (also shown in Figure 3). The special case when both strains are identical, $\sigma_{1}^{*}=\sigma_{2}^{*}=\sigma^{*}$, is implicit in Figure $3 . \Re_{2}<f\left(\Re_{1}\right)$ is a necessary condition for the stability of strain 1 (either a stable boundary endemic equilibrium $E_{1}$ or the equilibrium associated with strain-1 oscillations). Hence, $E_{1}$ is unstable when $\Re_{2}>f\left(\Re_{1}\right)$. Similarly, $E_{2}$ is unstable when $\Re_{1}>g\left(\Re_{2}\right)$. Hence, coexistence is expected when $\Re_{2}>f\left(\Re_{1}\right)$ and $\Re_{1}>g\left(\Re_{2}\right)$.

Next, the cases $\sigma_{2}^{*}<\sigma<\sigma_{1}^{*}$ and $\sigma_{1}^{*}<\sigma<\sigma_{2}^{*}$ are considered. $f\left(\Re_{1}\right)$ and $g\left(\Re_{2}\right)$ are increasing and decreasing functions of $\sigma$ correspondingly for $\sigma_{2}^{*}<\sigma<\sigma_{1}^{*}$ and decreasing and increasing (respectively) for $\sigma_{1}^{*}<\sigma<\sigma_{2}^{*}$ (Figure 4(b)). Hence, the stability region for strain 1 (Figure 4 (a), region $I$ ) may be significantly larger than that of strain 2 (Figure $4(\mathrm{a})$, region $I I$ ) for $\sigma_{2}^{*}<\sigma<\sigma_{1}^{*}$. For $\sigma_{1}^{*}<\sigma<\sigma_{2}^{*}$, the stability region of strain 1 may be noticeably smaller than that of strain 2 . The changes in the relative sizes of these stability regions seem to cause strong cross-immunity when it is conferred by strain $i\left(\sigma_{i} \downarrow 0\right)$ against an infection with strain $j$ (largely reduced susceptibility to alternative strain infections). The possibility that strain $j$ 

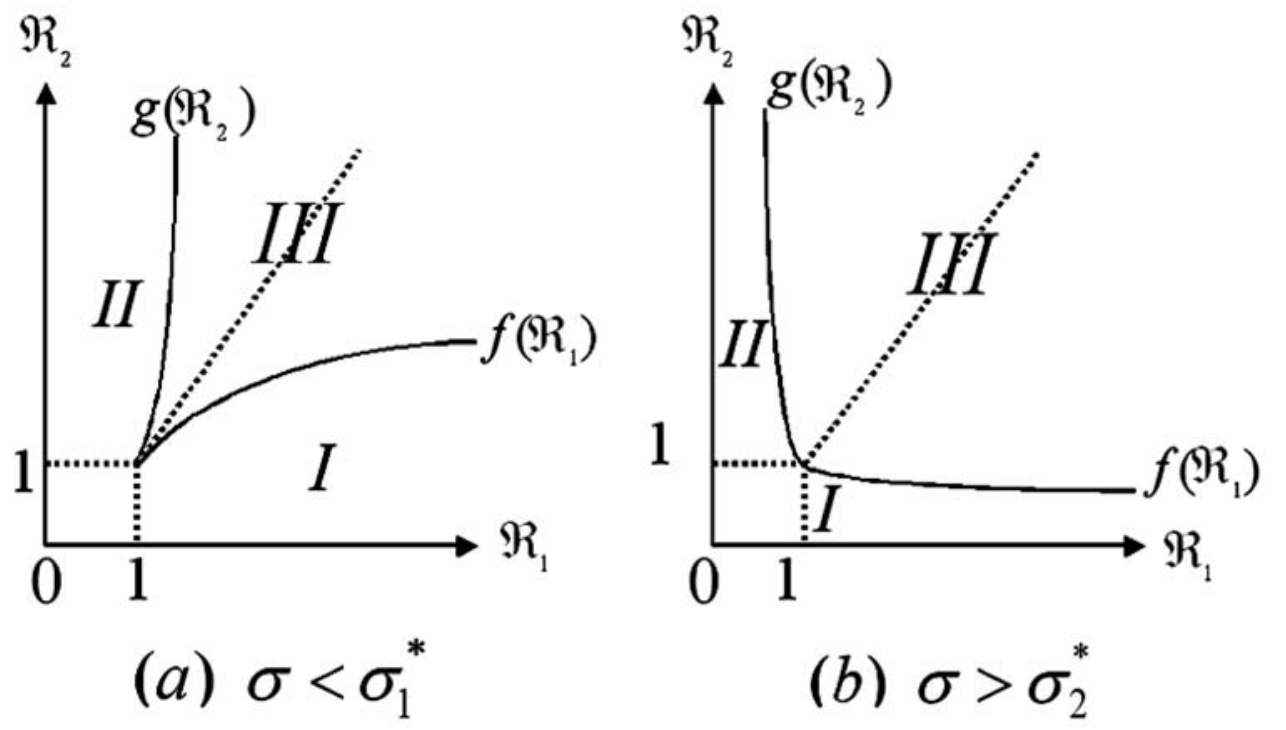

FIG. 3. Bifurcation diagram in the $\left(\Re_{1}, \Re_{2}\right)$-plane. The curves $\Re_{2}=f\left(\Re_{1}\right)\left(\right.$ for $\left.\Re_{1}>1\right)$ and $\Re_{1}=g\left(\Re_{2}\right)$ (for $\Re_{2}>1$ ) divide the region $\mathbf{R}_{+}^{2}-\left\{\left(\Re_{1}, \Re_{2}\right) \mid \Re_{1}<1\right.$ and $\left.\Re_{2}<1\right\}$ into three subregions: $I, I I, I I I$. When the parameters are in region $I$ (II), only strain 1 (strain 2) will be maintained (a stable boundary equilibrium or sustained oscillations of a single strain). In region III, both strains will be maintained (a stable boundary equilibrium or sustained oscillations of both strains).
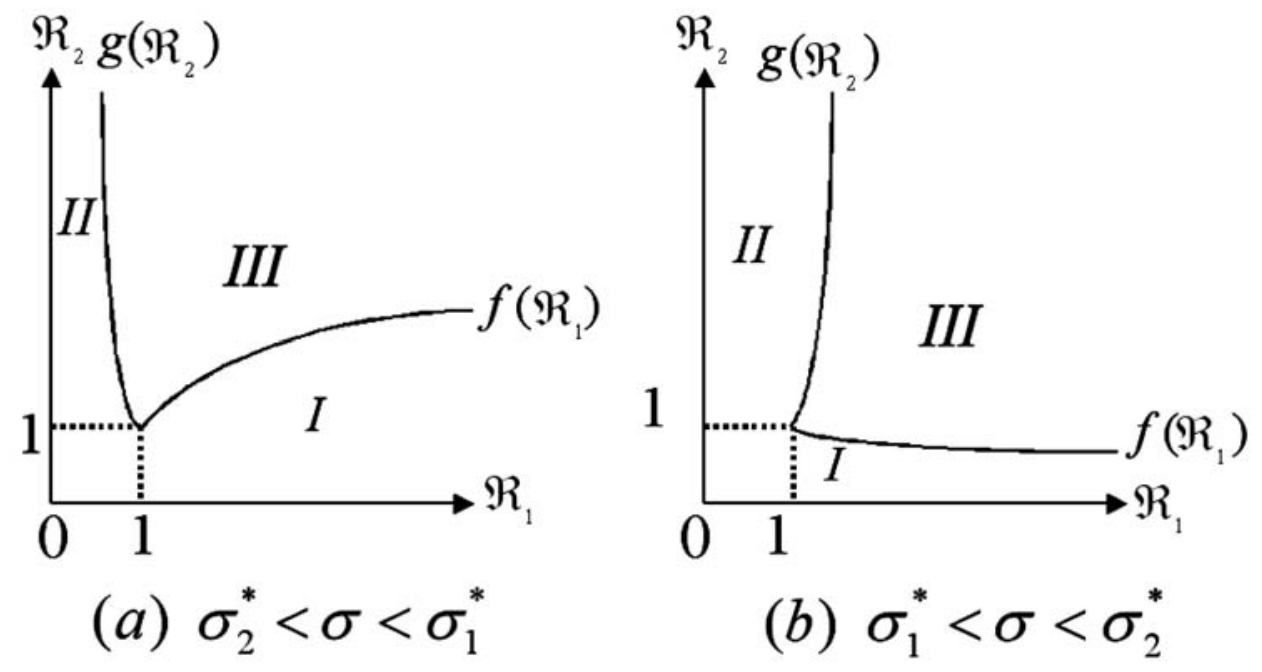

FIG. 4. Bifurcation diagram in the $\left(\Re_{1}, \Re_{2}\right)$-plane for the case when $\sigma_{1}^{*} \neq \sigma_{2}^{*}$. The curves $\Re_{2}=f\left(\Re_{1}\right)$ (for $\Re_{1}>1$ ) and $\Re_{1}=g\left(\Re_{2}\right)$ (for $\Re_{2}>1$ ) divide the region $\mathbf{R}_{+}^{2}-\left\{\left(\Re_{1}, \Re_{2}\right) \mid \Re_{1}<\right.$ 1 and $\left.\Re_{2}<1\right\}$ into three subregions: I, II, III. The meanings of these regions are the same as those in Figure 3. 

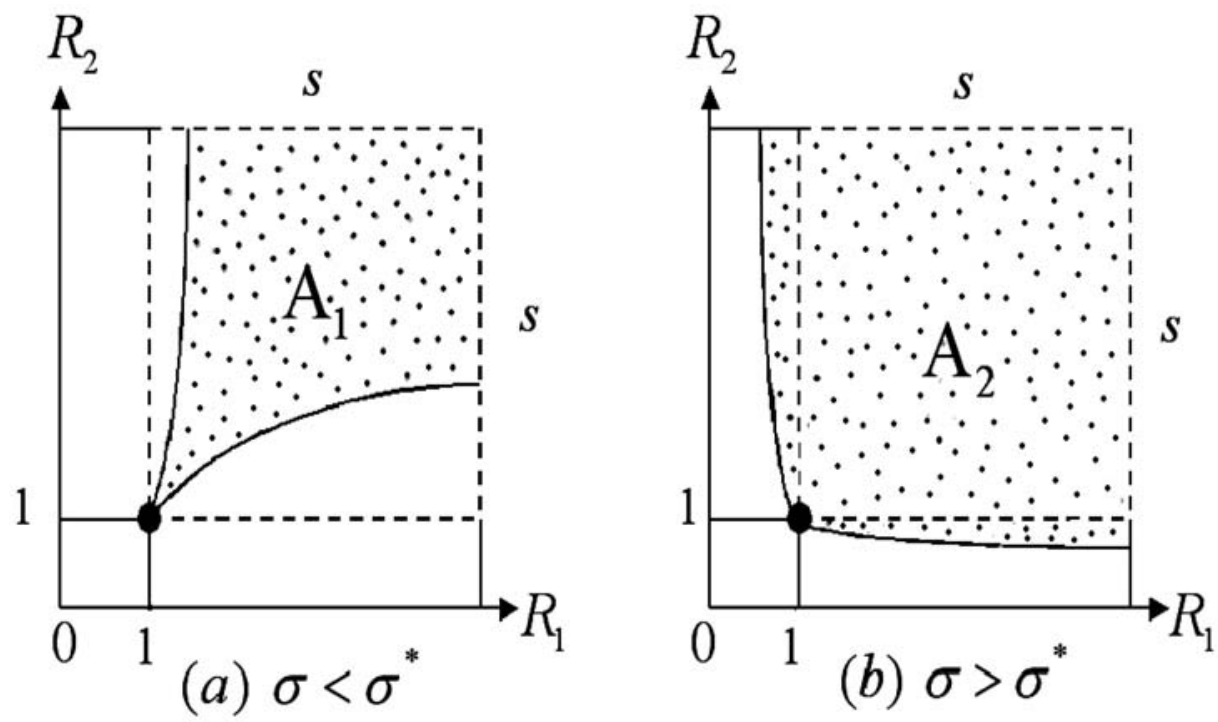

FIG. 5. $A_{1}$ and $A_{2}$ are the regions used to approximate the areas pertaining to section III in Figures 2(a)-(b). In order to approximate these areas we enclose regions $A_{1}$ and $A_{2}$ with a square with dimensions $s$ and corresponding area $s^{2}$ (dashed region); $s$ is chosen so that $1+s=\Re_{1}$ (and $\Re_{2} \approx \Re_{1}$ ) using the parameters in Table 1 and two values of $\sigma^{*}$ (see the text).

may become established under these conditions can be small. Likewise, weaker levels of cross-immunity to strain $j$ after an infection with strain $i\left(\sigma_{i} \uparrow 1\right)$ will support relatively larger regions of stability for strain $j$.

The stability regions for strain $1(I)$ and strain $2(I I)$ in the $\left(\Re_{1}, \Re_{2}\right)$-plane $\left(\sigma<\sigma_{1}^{*}\right.$ and $\left.\sigma>\sigma_{2}^{*}, \sigma_{1}^{*}=\sigma_{2}^{*}=\sigma^{*}\right)$ are illustrated in Figures 3(a)-(b). We show that as the levels of cross-immunity decrease, that is, as the values of $\sigma$ get closer to 1 (from Figure 3(a) to Figure 3(b)), the region of stability corresponding to each individual strain is reduced significantly (regions $I$ and $I I$ ). Simultaneously, an increase in the region of multiple strain coexistence $(I I I)$ can be observed as cross-immunity is weakened. It seems that as strains become antigenically distinct; that is, when cross-immunity against each other is weak, coexistence is more likely. Strong levels of cross-immunity ( $\sigma \downarrow 0)$ support the survival of a single strain; that is, in this case competition for susceptibles between strains is "fierce" ("competitive exclusion"). The strain with the highest ability to invade the host (largest $\Re_{q}$ ) is the most likely to become established (driving the other strain to extinction [4]).

Using Figures 5(a)-(b), a rough estimate for the "probability" of multiple strain coexistence is computed as a function of cross-immunity. The areas of both regions $A_{1}$ and $A_{2}$ (previously depicted by region $I I I$ ) by delineating the regions of interest with functions $f\left(\Re_{1}\right)$ and $g\left(\Re_{2}\right)$ are "approximately" computed. The area of $A_{1}$ in Figure $5(\mathrm{a})$ is enclosed by a square region with dimensions $s$, where $1+s=\Re_{1}$ and $\Re_{2} \approx \Re_{1}$. Similarly, the area $A_{2}$ in Figure $5(\mathrm{~b})$ is estimated. The selected value of $\sigma^{*}\left(\sigma^{*}=0.33\right)$ used corresponds to the one derived using the parameters provided in Table 1. A value of $\sigma=0.0008$ is used in Figure 5(a) and $\sigma=0.8$ in Figure 5(b). Letting $A_{1}(\sigma=0.0008)$ and $A_{2}(\sigma=0.8)$ in Figures $5(\mathrm{a})-(\mathrm{b})$ be the calculated areas corresponding to region $I I I$, we find that the quotient $A_{1} / A_{2}$ is small (0.0055562). 

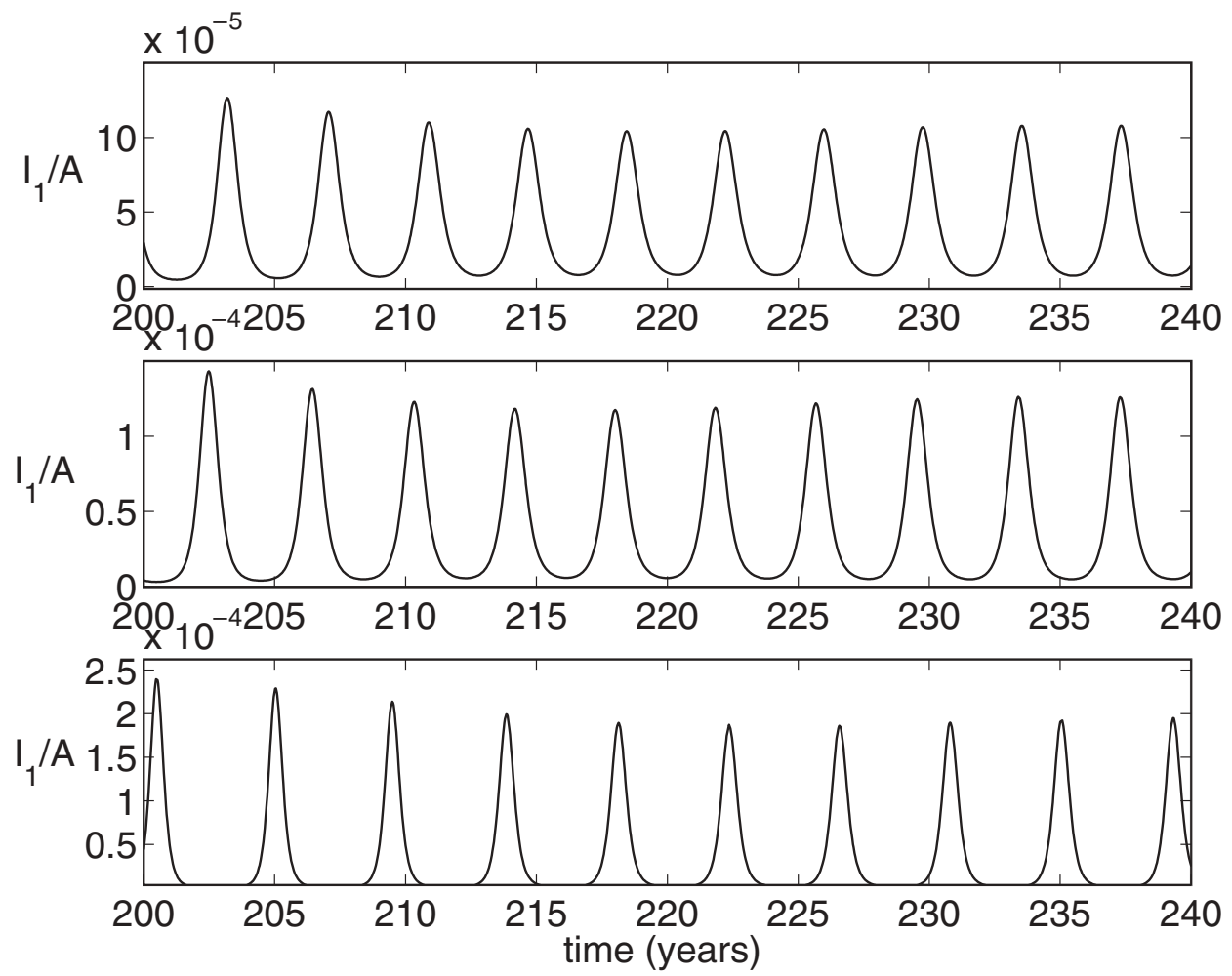

FIG. 6. Numerical integration of the model equations. The fraction of the infective individuals (nonisolated) with strain $1\left(I_{1} / N\right)$ is shown for increasing periods of isolation. The length of the isolation period has been chosen (from top to bottom) to be 3 days, 7 days, and 15 days. Crossimmunity between strains is intermediate $(\sigma=0.5)$.

Hence, the coexistence of antigenically similar strains (sharing strong levels of crossimmunity) seems less likely than when cross-immunity is weak.

5. Numerical results. In this section we explore the model equations numerically as the levels of cross-immunity and isolation are varied. In the first set of simulations, we study the symmetric case $\left(\sigma_{12}=\sigma_{21}=\sigma\right)$. We explore the role of crossimmunity and host isolation in supporting sustained oscillations for a single and/or both strains where $\sigma \in(0.01,0.8)$ and $\frac{1}{\alpha}$ is either 1 day, 3 days, or 15 days. In the second set of simulations, we explore the case where $\sigma_{12} \neq \sigma_{21}$. Average life expectancy is fixed at 70 years; infected individuals recover from infection in 5-7 days; individuals are isolated for 1-15 days. The parameters used in simulations are listed in Table 1.

Case 1. $\sigma_{12}=\sigma_{21}=\sigma$. The robustness of multiple strain coexistence begins from the assumption that both strains are present in the population $(s(0)=0.4$, $i_{1}(0)=0.199, r_{1}(0)=r_{2}(0)=0.2, i_{2}(0)=0.001$, and $\left.q_{1}(0)=q_{2}(0)=w(0)=0\right)$. Simulations are conducted using varying levels of cross-immunity $(\sigma=0.01,0.33$, 0.5 , and 0.8 ) and isolation periods (1 day, 3 days, and 15 days). Figure 6 shows that for intermediate cross-immunity $(\sigma=0.5)$ the periods between outbreaks is approximately 4 years with an amplitude ranging from $1.1 \times 10^{-4}$ to $1.5 \times 10^{-4}$. Figure 7 shows that strong cross-immunity gives periods of approximately 3 years and amplitude of $2 \times 10^{-4}$. As the levels of cross-immunity range from intermediate 


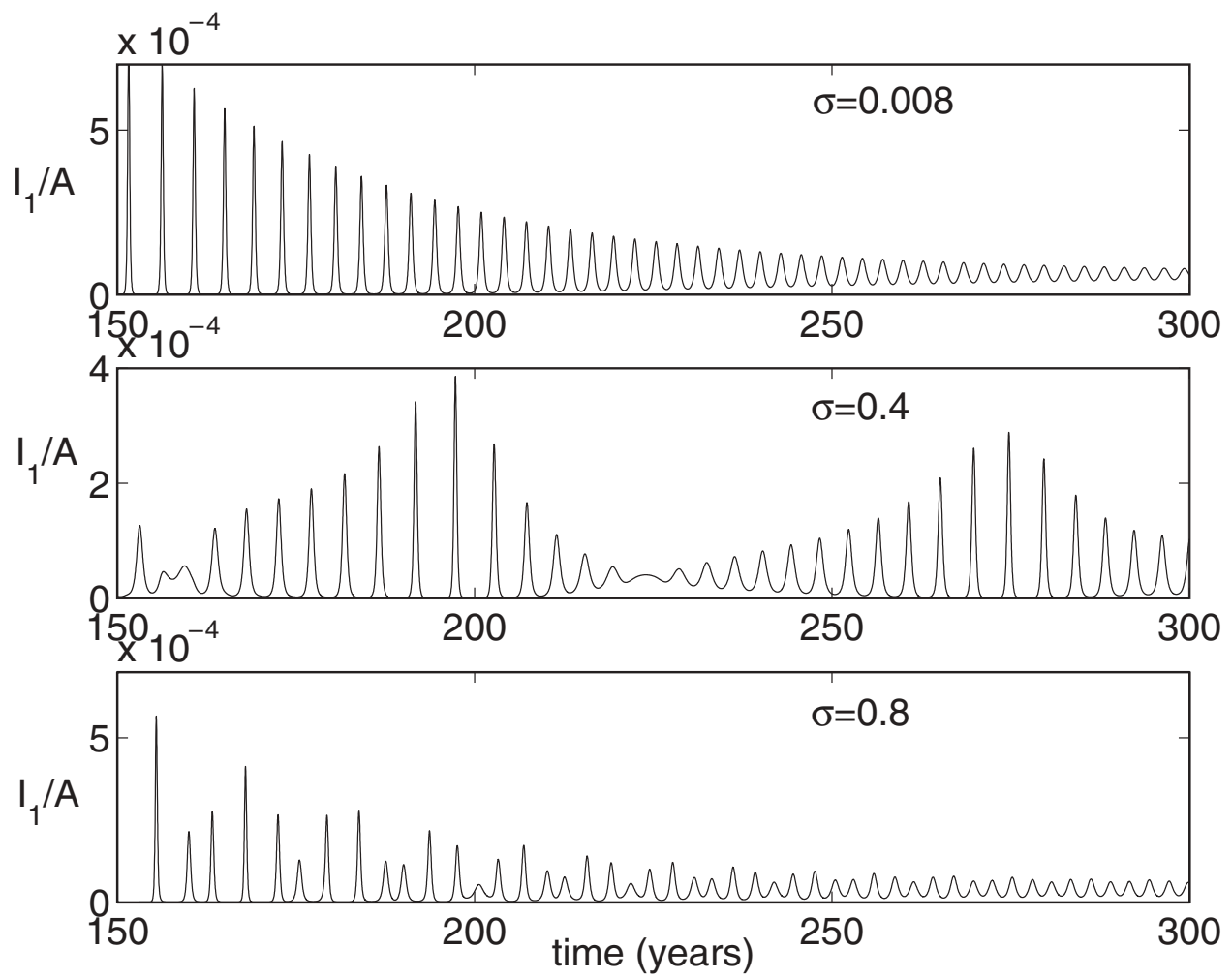

FIG. 7. Numerical integration of the model equations. The fraction of the infective individuals (nonisolated) with strain $1\left(I_{1} / N\right)$ is shown. The isolation period is fixed at 3 days, while crossimmunity levels are varied (from top to bottom): 0.008 (strong), 0.4 (intermediate), and 0.8 (weak).

to weak, the periods become more irregular, and the amplitude ranges vary $\left(4 \times 10^{-5}\right.$ to $4 \times 10^{-4}, \sigma=0.4$ and $\left.1-5.5 \times 10^{-4}, \sigma=0.8\right)$.

Case 2. $\sigma_{12} \neq \sigma_{21}$. Briefly, we study the effect of isolation for asymmetric crossimmunity by allowing strains to become antigenically distinct with increasing $\varepsilon$ (that is, $\left|\sigma_{12}-\sigma_{21}\right|=\varepsilon$ ) (see also [12]). We assume a 3-day isolation period. Figure 8 illustrates the interactions that arise between nonsymmetric strains as their difference in cross-immunity increases, $\varepsilon \in(0.01,0.03)$. The periods between oscillations for strain 1 (solid) vary from 10-11 years with increasing $\varepsilon$ and decreasing levels of cross-immunity. Similarly, the periods between oscillations corresponding to strain 2 (dashed) vary from 10-13 years. The amplitude with highest peak for strain 1 (3.8 $\times$ $\left.10^{-4}\right)$ is attained at $\varepsilon=0.02$, whereas that of strain 2 is observed at $\varepsilon=0.02$ and $\varepsilon=0.03\left(3 \times 10^{-4}\right)$. Figure 8 shows that for intermediate coupled strains $\left(\sigma_{i j} \approx 0.33\right)$, the system goes through cycles with approximate periods of 10-13 years, where each cycle may contain minor outbreaks followed by a period with very low disease levels $\left(1-2 \times 10^{-5}\right)$.

6. Discussion. "Flu" epidemic patterns include yearly outbreaks (antigenic drift), the explosive onset of outbreaks, the rapid termination of local epidemics (despite an "abundance" of susceptible individuals), and potentially major pandemics (antigenic shift). The continuous generation (most likely from random mutations in the NS gene) of new "flu" strains ("minor" genetic changes) and the sudden generation of subtypes (radical genetic changes) and their impact on the history of 


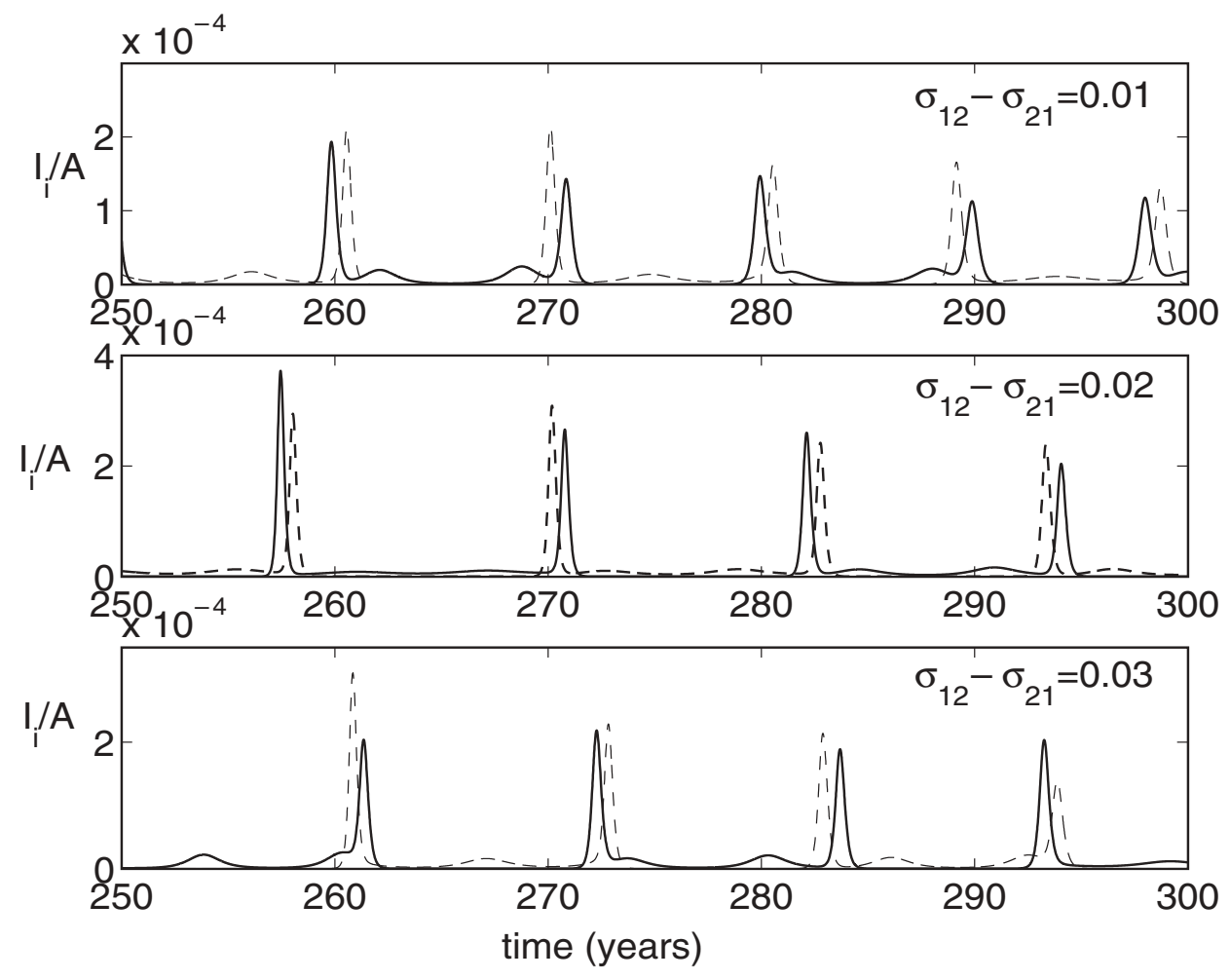

FIG. 8. Numerical integration of the model equations. The fraction of the infective individuals (nonisolated) with strain 1 (solid) and strain 2 (dashed) is shown. Differences in cross-immunity levels between strains 1 and $2\left(\sigma_{12}-\sigma_{21}\right)$ increase (from top to bottom): 0.01, 0.02, and 0.03. For example, cross-immunity for strains 1 and 2 correspondingly are given by $\sigma_{12}=0.36$ and $\sigma_{21}=0.33$ (bottom panel).

acquired (age-dependent) immunity of host populations make the study of influenza dynamics and its control challenging and fascinating $[2,16]$.

The focus of this article is on the time evolution of influenza $\mathrm{A}$ in a nonfixed landscape driven by tight coevolutionary interactions (that is, interactions where the fate of the host and the parasite are intimately connected; see [25]) between human hosts and competing strains. The process is mediated by intervention (behavioral changes) and cross-immunity. In other words, the nature of the invading landscape (susceptible host) changes dynamically from behavioral changes (isolation, short time scale) and past immunological experience (cross-immunity, long time scale).

The "partial" herd-immunity generated by past history of invasions on the host population can have a huge impact on the quantitative dynamics of the "flu" at the population level. The assumption that $\sigma_{12}=\sigma_{21}=\sigma$ for $i \neq j$ naturally results in a dynamic landscape that is not too different (in the oscillatory regime) than the one observed on single-strain models with isolation $[15,20]$. That is, a lack of heterogeneity in cross-immunity results in a system "more or less" driven (in the oscillatory regime) by the process of isolation. However, small variations in isolation (Figure 6) leads to radically quantitatively distinct epidemics in the oscillatory regime. This modeling framework (see also $[5,6]$ ) can "asses" the impact of antigenically similar $\left(\left|\sigma_{i j}-\sigma_{j i}\right| \rightarrow 0\right)$ and antigenically distinct strains $\left(\left|\sigma_{i j}-\sigma_{j i}\right|>\epsilon\right)$. 
In all cases, sustained oscillations with periods that are consistent with influenza epidemics/pandemics are possible [11,28]. These results are consistent with those obtained in single-strain models [14] (i.e., sustained oscillations are preserved), except that the oscillations are now possible for "realistic" isolation periods. The introduction of a second strain enhances the possibilities. Numerical simulations illustrate various outcomes, including competitive exclusion, coexistence, and subthreshold coexistence. The interepidemic periods range from 2 to 10-13 years, depending on the levels of cross-immunity. Strong intermediate asymmetric cross-immunity leads to interepidemic periods in the range of 10-13 years. Symmetric cross-immunity reduces the range to 1-3 years. The results of intermediate (symmetric) cross-immunity are consistent with those found in $[5,6]$. Documented evidence on the cocirculation of strains belonging to the same subtype $[11,28]$ appears to be consistent with these results.

Our results show that multiple strain coexistence is highly likely for antigenically distinct (weak cross-immunity) strains and not for antigenically similar under symmetric cross-immunity ("competitive exclusion" principle [4]). As the levels of cross-immunity weaken, the likelihood of subthreshold coexistence $\left(\Re_{i}^{j}<1\right)$ increases. However, "full" understanding of the evolutionary implications that result from human host and influenza virus interactions may require the study of systems that incorporate additional mechanisms such as seasonality in transmission rates, age-structure, individual differences in susceptibility or infectiousness, and the possibility of coinfections. Thacker [28] notes that the observed seasonality of influenza in temperate zones may be the key to observed patterns of recurrent epidemics. Superinfection may also be a mechanism worth consideration, even though studies in [22] show that it is only moderately possible for young individuals to become infected with two different strains in one "flu" season.

The recent flu epidemic [3] which has invaded all 50 states (2003-2004) and our experiences with the recent SARS epidemic [10] are a source of concern. While isolation and quarantine [8] seem effective [10], they can "destabilize" "flu" dynamics (oscillations) and generate some level of uncertainty. The results in this paper suggest the need to explore the long-term impact of current U.S. vaccination policies on the levels of cross-immunity generated by herd-immunity in the case of the flu. Whether or not they increase or reduce the likelihood of a future major outbreak is a question worth considering.

Appendix A. The local stability of the disease-free state follows from the study of the eigenvalues of the Jacobian matrix $J$ of system (1) at $E_{0}$. The $10 \times 10$ Jacobian matrix $J$ is partitioned after arranging the variables, that is, after rewriting system (1) as $d M / d t=F(M)$, where $M=\left(S, I_{1}, I_{2}, Q_{1}, Q_{2}, R_{1}, R_{2}, V_{1}, V_{2}, W\right)$. The corresponding eigenvalues are given by $\lambda_{i}=-\mu$ for $i=1,2,3,4 ; \lambda_{i}=\beta_{i}-\left(\mu+\gamma_{i}+\delta_{i}\right)$ for $i=1,2 ; \lambda_{i}=-\left(\mu+\alpha_{i}\right)$ for $i=1,2 ; \lambda_{i}=\left(\mu+\gamma_{i}\right)$.

Acknowledgments. The authors are sincerely grateful to Gerardo Chowell, Laura Jones, Prasith Baccam, Catherine Macken, and Christopher Kribs for their beneficial comments and suggestions. The authors would also like to thank LANL (Center for Nonlinear Studies) for their support during the visit of Dr. Castillo-Chavez as a Ulam Scholar.

\section{REFERENCES}

[1] V. Andreasen, J. Lin, And S. A. Levin, The dynamics of co-circulating influenza strains conferring partial cross- immunity, J. Math. Biol., 35 (1997), pp. 825-842. 
[2] azcentral.com, Flu Is Confirmed in All 50 States; Vaccine Dwindles, http://www.azcentral. $\mathrm{com} /$ news/articles/1213flu-outbreak13.html (2003).

[3] D. A. Buonagurio, S. Nakada, J. D. Parvin, M. Krystal, P. Palese, and W. M. Fitch, Evolution of human influenza A viruses over 50 years: Rapid, uniform rate of change in NS gene, Science, 232 (1986), pp. 980-982.

[4] H. J. Bremermann and H. R. Thieme, A competitive exclusion principle for pathogen virulence, J. Math. Biol., 27 (1989), pp. 179-190.

[5] C. Castillo-Chavez, H. W. Hethcote, V. Andreasen, S. A. Levin, and W. M. Liu, Crossimmunity in the dynamics of homogeneous and heterogeneous populations, in Mathematical Ecology, World Scientific, Teaneck, NJ, 1988, pp. 303-316.

[6] C. Castillo-Chavez, H. W. Hethcote, V. Andreasen, S. A. Levin, and W. M. Liu, Epidemiological models with age structure, proportionate mixing, and cross-immunity, J. Math. Biol., 27 (1989), pp. 233-258.

[7] C. Castillo-Chavez and H. R. Thieme, Asymptotically autonomous epidemic models, Mathematical Population Dynamics: Analysis of Heterogeneity, Vol. 1: Theory of Epidemics, O. Arino, D. Axelrod, M. Kimmel, and M. Langlais, eds., Wuerz, Winnepeg, ON, Canada, 1995, pp. 33-50.

[8] C. Castillo-Chavez, C. W. Castillo-Garsow, and A. A. Yakubu, Mathematical models of isolation and quarantine, JAMA, 290 (2003), pp. 2876-2877.

[9] CDC (Centers for Disease Control), The Influenza (Flu) Viruses, http://www.cdc.gov/ncidod/ diseases/flu/viruses.htm (2003).

[10] G. Chowell, P. W. Fenimore, M. A. Castillo-Garsow, and C. Castillo-Chavez, SaRS outbreak in Ontario, Hong Kong and Singapore: The role of diagnosis and isolation as a control mechanism, J. Theor. Biol., 24 (2003), pp. 1-8.

[11] R. B. Couch And J. A. Kasel, Immunity to influenza in man, Ann. Rev. Micro., 31 (1983), pp. 529-549.

[12] J. H. P. Dawes And J. R. GoG, The onset of oscillatory dynamics in models of multiple disease strains, J. Math. Biol., 45 (2002), pp. 471-510.

[13] K. Dietz, Epidemiological interference of virus populations, J. Math. Biol., 8 (1979), pp. 291300.

[14] Z. Feng, Multi-Annual Outbreaks of Childhood Diseases Revisited the Impact of Isolation, Ph.D. thesis, Arizona State University, Tempe, AZ, 1994.

[15] Z. Feng And H. R. Thieme, Recurrent Outbreaks of childhood diseases revisited: The impact of isolation, J. Math. Biosci., 128 (1995), pp. 93-130.

[16] W. M. Fitch, R. M. Bush, C. A. Bender, And N. J. Cox, Long term trends in the evolution of H(3) HA1 human influenza type A, Proc. Nat. Acad. Sci. U.S.A., 94 (1997), pp. 7712-7718.

[17] J. P. Fox, C. E. Hall, M. K. CoOneY, AND H. M. Foy, Influenza virus infections in Seattle families, 1975-1979, Amer. J. of Epidemiology, 116 (1982), pp. 212-227.

[18] W. H. Hethcote and S. A. Levin, Periodicity in epidemiological modeling, in Applied Mathematical Ecology, Biomathematics 18, Springer-Verlag, Berlin, 1989, pp. 193-211.

[19] H. W. Нетнсоте, The mathematics of infectious diseases, SIAM Rev., 42 (2000), pp. 599-653.

[20] W. H. Нетhсоте, M. Zhien, And L. Shengbing, Effects of quarantine in six endemic models for infectious diseases, Math. Biosci., 180 (2002), pp. 141-160.

[21] M. K. Holmes, Introduction to Pertubation Methods, Springer-Verlag, New York, 1995.

[22] R. E. Hope-Simpson, Epidemic mechanisms of Type A influenza, J. Hyg. Camb., 83 (1979), pp. 11-26.

[23] T. KATO, Perturbation Theory for Linear Operators, Springer-Verlag, Berlin, 1976.

[24] R. M. KRug, The Influenza Viruses, Plenum Press, New York, 1989.

[25] S. A. Levin And C. Castillo-Chavez, Topics in evolutionary biology, in Mathematical and Statistical Developments of Evolutionary Theory, NATO Adv. Sci. Inst. Ser. C Math. Phys. Sci. 299, S. Lessard, ed., Kluwer Academic Publishers, Dordrecht, Boston, London, 1990, pp. 327-358.

[26] J. Lin, V. Andreasen, And S. A. Levin, Dynamics of influenza A drift: The linear three-strain model, J. Math. Biosci., 162 (1999), pp. 33-51.

[27] L. H. Taber, A. Paredes, W. P. Glezen, and R. B. Couch, Infection with influenza A/Victoria virus in Houston families, 1976, J. Hyg. Cam., 86 (1981), pp. 303-313.

[28] S. B. Thacker, The persistence of influenza in human populations, Epidemi. Rev., 8 (1986), pp. 129-142.

[29] R. G. Webster, W. J. Beam, O. T. Gorman, T. M. Chambers, and Y. Kawaoka, Evolution and ecology of influenza A viruses, Micro. Biol. Rev., 56 (1992), pp. 152-179. 